\title{
Gestión de proyectos de sistemas de información. Conectando el agile mindset, la motivación y el liderazgo para la generación de equipos de alto desempeño
}

\author{
Marco Antonio Fernández del Pomar \\ marco9129@gmail.com / Universidad de Lima. Lima, Perú
}

Recepción: 22-6-2018 / Aceptación: 20-8-2018

Resumen. El presente artículo describe los pilares fundamentales para la generación de equipos de alto desempeño dentro del ámbito de la gestión de proyectos de sistemas de información. Se describirá cómo la adopción del agile mindset, la comprensión de la evolución de la motivación y la interiorización del costo del liderazgo permiten transformar el desempeño de cualquier equipo de personas.

PALABRAS CLAVE: gestión de proyectos, tecnologías de información, sistemas, liderazgo, equipos, alto desempeño, motivación, ágil, marcos de trabajo ágiles, mindset

\section{Information systems project management.}

Connecting agile mindset, motivation and leadership for the creation of high-performance equipment

Abstract. This paper describes the fundamental pillars for the generation of high-performance teams within the scope of the management of projects of information systems. It will be described how the adoption of the Agile Mindset, the understanding of the evolution of motivation and the internalization of the cost of leadership allows to transform the performance of any team of people.

KEYWORDS: project management, information technology, systems, leadership, teams, high performance, motivation, agile, agile methodologies, mindset 


\section{INTRODUCCIÓN}

Han pasado ya 17 años desde la firma del Agile Manifiesto en el 2001. No obstante, existen aún muchas organizaciones que están recién aprendiendo a adoptar marcos de trabajo ágiles para la gestión de sus proyectos. Y, a pesar de existir infinidad de documentación, casos de éxito, información en la web y frameworks libres como SAFe, existen aún múltiples casos de empresas cuyo principal obstáculo para implementar de forma adecuada un marco de trabajo ágil es su propia cultura organizacional.

No es novedad que la cultura de una organización puede jugar en contra al momento de tratar de implementar cambios organizacionales de alto impacto, como es migrar de una mentalidad de gestión de proyectos tradicional hacia una ágil. Sin embargo, la problemática de fondo va más allá de la cultura de la empresa y tiene que ver con aspectos tan fundamentales como una correcta comprensión de la motivación y el liderazgo en nuestras organizaciones.

Existe abundante literatura sobre la motivación de equipos, al igual que sobre liderazgo. A pesar de esto existen aún vacíos parciales sobre estos dos conceptos, los cuales abordaremos para lograr completar la visión necesaria para generar equipos de alto desempeño. Para lograr este objetivo se requiere conocer de dónde viene esta necesidad de motivar a las personas para poder entender cómo aplicar de forma óptima las buenas prácticas de gestión de equipos. De igual forma, es imperativo conocer el aspecto del liderazgo que se ha visto sesgado en un aspecto crítico de su definición; este aspecto es el costo del liderazgo. Este sesgo o limitante en la definición de liderazgo puede ocasionar impactos negativos imprevistos no identificados al momento de gestionar un grupo humano. El impacto de no considerar la visión completa del liderazgo que describiremos a lo largo de este artículo es una de las razones por las que muchas organizaciones batallan para generar el cambio de mindset y no logran migrar de forma óptima hacia aquellas prácticas ágiles a nivel corporativo que les permitan generar equipos de alto desempeño.

Por supuesto, no es suficiente con comprender los conceptos planteados en los párrafos anteriores. Es necesario alinearlos con aquellos principios y valores ágiles que no solo son fundamentales para aplicar marcos de trabajo ágiles en la gestión de proyectos, sino que son críticos para llevar a los equipos de proyectos a niveles de desempeño superiores. Esta alineación permite no solamente poder realizar una suave transición hacia un agile mindset, sino también facilita a aquellas organizaciones que ya tienen implementada una cultura ágil a optimizar las buenas prácticas de motivación para sus equipos de trabajo y desarrollar lideres con una visión más amplia y sin ningún sesgo en sus prácticas de liderazgo.

\section{GENERACIÓN DE EQUiPOS DE ALTO DESEMPEÑo}

Actualmente instituciones como el Project Management Institute (PMI), Scaled Agile Framework (SAFe) o Axelos, son las que lideran el mercado en cuanto a guías de buenas 
prácticas, marcos de trabajo y metodologías para la gestión de proyectos y temas relacionados. Aquellos que han leído a fondo las principales publicaciones de estas instituciones se darán cuenta de que, a pesar de que estas pueden tener diferentes acercamientos al ámbito de gestión de proyectos y a qué nivel se aplica, tienen en común muchos aspectos respecto al manejo de equipos de proyecto. Específicamente en los siguientes puntos:

- El empoderamiento de los equipos.

- La ejecución de las tareas y actividades propias del proyecto de una forma sostenible en el tiempo y sin desgastar al equipo.

- Mantener y promover un liderazgo adecuado para el equipo.

\subsection{Valores del Manifiesto ágil}

Los aspectos mencionados se encuentran altamente relacionados con el primer valor del Manifiesto ágil, que declara que los "Individuos e interacciones [están] sobre procesos y herramientas”. De este valor se desprenden dos principios:

- Construya proyectos en torno de individuos motivados. Bríndeles el ambiente y soporte que necesitan y confíe en ellos para hacer el trabajo.

- Los procesos ágiles promueven el desarrollo sostenible. Los sponsors, desarrolladores, y usuarios deben poder mantener un ritmo constante indefinidamente.

Son las personas las que ejecutan los proyectos y hacen realidad el valor generado por estos para las organizaciones. Los escritores del Manifiesto ágil identificaron el valor de tener un equipo de personas altamente motivadas e involucradas con el proyecto. Lo cual nos lleva a la pregunta de por qué las organizaciones, 17 años después del Manifiesto ágil, aún no tienen del todo claras las consideraciones que se deben tener en cuenta para generar estos equipos superiores. No es la falta de conocimiento sobre los conceptos de motivación y liderazgo sino el desconocimiento de aspectos fundamentales sobre estos lo que ocasiona que muchas veces, al aplicar técnicas o prácticas de motivación y liderazgo, fracasen rotundamente.

Para empezar a entender cómo es que la conexión entre el Manifiesto ágil, la evolución de la motivación y el costo del liderazgo nos puede ayudar a generar equipos de alto desempeño, debemos entender cada uno de estos conceptos y cómo es que están relacionados.

Comencemos por entender un aspecto poco conocido sobre la motivación: su evolución. Pink (2011) explica en su libro Drive, cómo es que hemos pasado de una motivación 1.0, donde el ser humano solo buscaba aspectos de motivación biológica, tales como comer, dormir y sobrevivir, a una motivación 2.0, la cual surgió con la creación del dinero, que se utiliza para recompensar una actividad específica (trabajo). La lógica de la motivación 2.0 es que recompensar una actividad te dará más de esta. Penalizar una actividad te dará menos de esta. 
Esta motivación, también llamada algorítmica, es dominada por el hemisferio izquierdo del cerebro y se caracteriza por relacionarse con la motivación extrínseca. Esta etapa de la evolución es la que existe aún hoy en día y su lógica es aplicada virtualmente en todos los ambientes laborales. Aún seguimos aplicando la misma lógica descrita anteriormente, a través de la cual invertimos tiempo en una actividad específica (trabajo) para recibir una recompensa extrínseca (dinero). Posteriormente Pink (2011) habla sobre la motivación 3.0, mejor conocida como motivación intrínseca, la cual es dominada por el hemisferio derecho del cerebro, el cual también es aquel que controla los aspectos emocionales y creativos de las personas. Es en esta etapa, la motivación 3.0, en la que nos enfocaremos.

Pero se preguntarán: ¿en qué se relaciona la evolución de la motivación con los equipos de alto desempeño? Pues es justamente la falta de comprensión de esta relación la base de la problemática de muchas organizaciones. Pink (2011) relaciona su teoría de evolución de la motivación con la famosa pirámide de necesidades de Maslow (1943). Es así como podemos completar mejor la relación entre la motivación y la generación de equipos superiores.

Todos los miembros del equipo y los seres humanos en general buscan alcanzar el último nivel de la pirámide, la autorrealización (self-actualization). La figura 1 muestra esta relación.

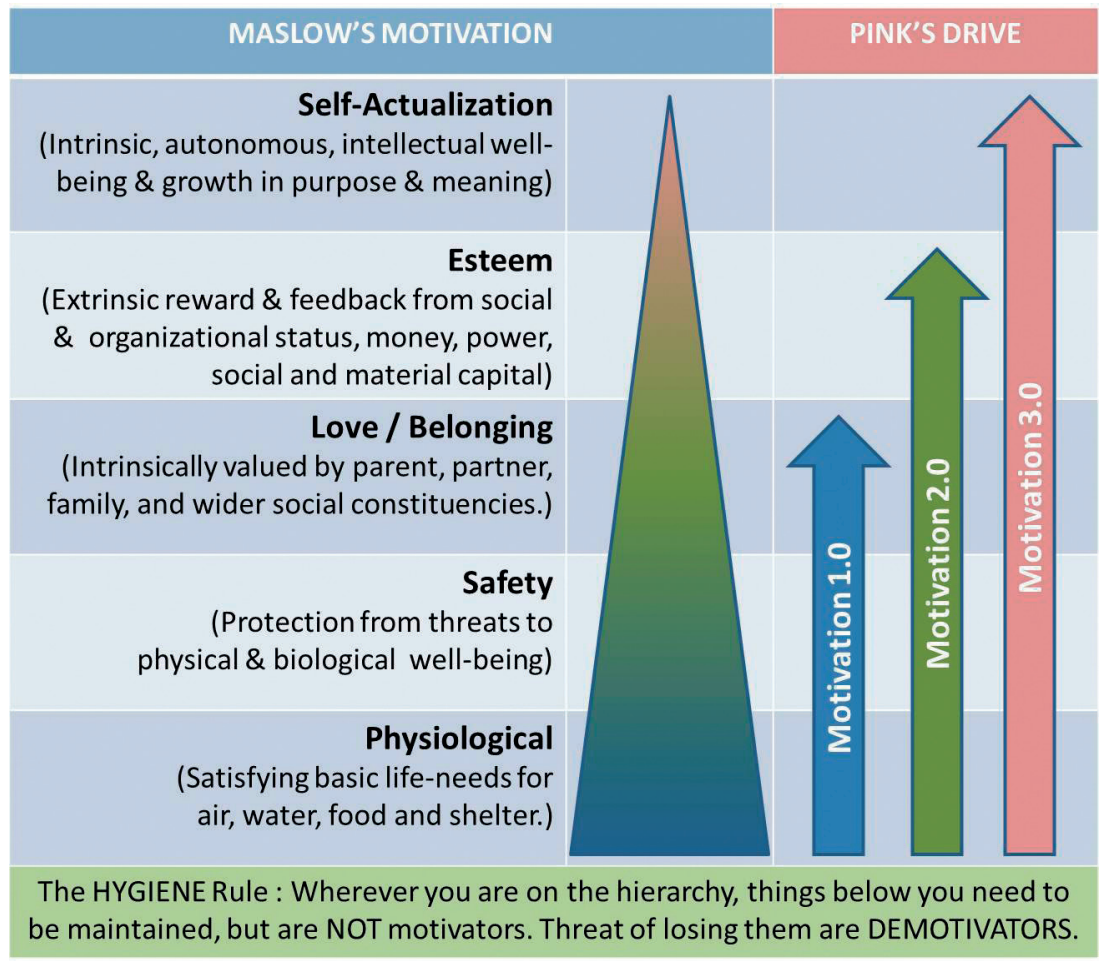

Figura 1. Relación entre teoría de evolución motivacional y pirámide de Maslow

Fuente: Psybertron Asks. www.psybertron.org/archives/5309 


\subsection{Motivación 3.0}

La motivación 3.0 es aquella que se sostiene en cuatro pilares: relación, autonomía, maestría y propósito (RAMP por sus siglas en inglés). Al comprender cada uno de estos pilares nos daremos cuenta de que se refieren a características descritas en la literatura de buenas prácticas de gestión de proyectos. Los equipos deben relacionarse entre ellos de forma óptima; ya que son equipos multidisciplinarios deben tener autonomía para trabajar libremente, deben tener maestría o dominio sobre sus funciones y deben tener un propósito claro. En la figura 2 se muestran las tres etapas de la evolución de la motivación (Pink, 2011).

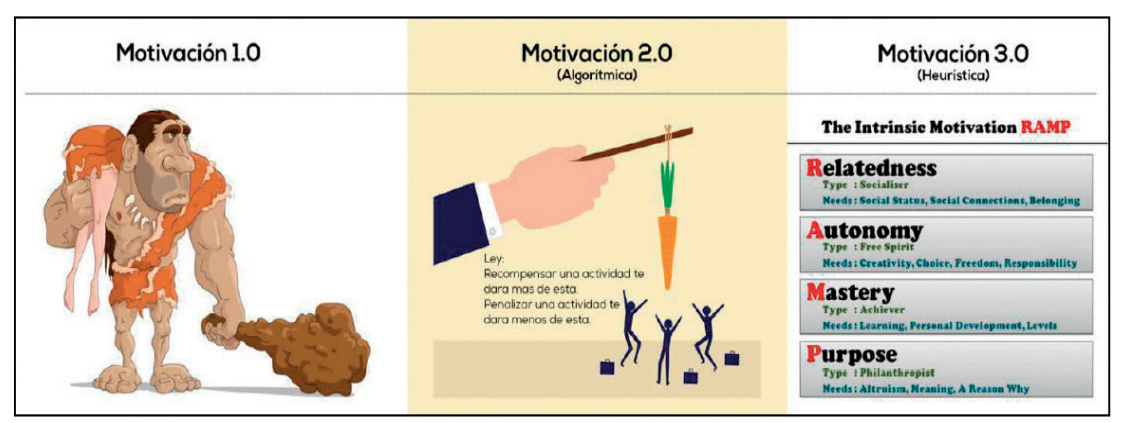

Figura 2. Las tres etapas de la evolución de la motivación

Elaboración propia

Pero si estas características se encuentran en la literatura de gestión de proyectos, ¿por qué es tan difícil generar equipos de alto desempeño? La respuesta es simple: la mayoría de las empresas mantienen el modelo de motivación $2.0 \mathrm{y}$, adicionalmente, la típica resistencia al cambio organizacional por parte de altos ejecutivos; el migrar al modelo 3.0 implica un cambio de mentalidad drástico de toda la organización.

El modelo actual promueve, en su mayoría, una motivación extrínseca, por lo que es difícil que los equipos alcancen los cuatro pilares de la motivación 3.0 (RAMP). Es decir, la motivación 2.0 solo se enfoca en el hemisferio izquierdo del cerebro, aquel que usa solo la lógica, mas no llega de forma óptima al hemisferio que controla las emociones y el sentido de propósito, el hemisferio derecho, el cual, cabe resaltar, es el responsable del lado creativo de los seres humanos, y lo más importante es de donde viene aquello que muchas empresas hoy en día desean obtener, la capacidad de que su gente innove.

\subsection{Liderazgo en equipos de alto desempeño}

Sin embargo, esta teoría revisada por sí sola no explica de forma completa la dificultad de generar equipos de alto desempeño. Aunque las necesidades de los miembros del equipo se 
vieran cubiertas y abarcaran el RAMP, aún falta una pieza fundamental: el liderazgo. De igual forma que con la teoría anterior, es necesario comprender un aspecto poco conocido del liderazgo, el costo que implica.

Sinek (2014), en su libro Leaders Eat Last, lo explica muy bien. El liderazgo tiene un costo, y uno del cual muchas veces no somos conscientes (o no queremos serlo). El líder debe estar preparado para sacrificarse por su equipo. Esto responde, al igual que lo afirmado por Pink (2011), a una teoría evolutiva histórica del Homo sapiens. Los seres humanos somos seres sociales, pero más que eso, nuestras sociedades son jerárquicas.

Hace miles de años, los líderes de las entonces tribus nómadas eran elegidos por rasgos muy específicos. Fuerza, velocidad, agresividad, entre otros. Como en muchas especies animales, el líder de la manada o tribu recibía mayores y mejores recompensas. Comía primero, comía mejor, elegía una (o varias) hembras para prolongar la especie, entre otros beneficios. Hoy en día, nuestras tribus han sido reemplazadas por organizaciones donde los líderes no se eligen por fuerza o velocidad sino por otras capacidades, como inteligencia emocional, conocimientos técnicos, facilidad de habla en público, empatía, etcétera.

A primera vista, para un líder, en cualquiera de las dos épocas, la vida era más simple y acogedora. Sin embargo, el liderazgo no venía gratis. La tribu esperaba mucho en retorno para dar ese ansiado título a quien supuestamente era más capaz. El líder no solo debía guiar a toda la tribu hacia la dirección correcta para sobrevivir en la época de las cavernas. Era responsable de proteger a todos los miembros, de tomar decisiones sobre qué cazar, cuándo, y cómo, de decidir cuándo era momento de migrar o de asentarse en un lugar, etcétera. Pero, sobre todo, se esperaba que, llegado el momento y de ser necesario, el líder se sacrificase por su tribu para salvarla de cualquier amenaza interna o externa. Es esta gran parte del liderazgo la que parece haberse extinguido en muchos de los líderes de hoy.

Ciertamente en nuestra época no nos referimos a sacrificar la vida de una persona si es que el equipo no cumplió con la fecha de entrega del proyecto, pero sí nos referimos a que el líder tome responsabilidad y proteja a cada miembro del equipo de cualquier amenaza externa o interna y cubra sus espaldas, asegurándose de que los miembros del equipo se sientan protegidos y seguros dentro de sus ambientes de trabajo. Esto obedece a una respuesta evolutiva de esperar que la persona liderándote dentro de tu tribu (empresa), se encargue de que tu enfoque sea solo trabajar eficientemente en tus funciones específicas sin tener que dedicar tiempo y energía a preocuparte por las amenazas que rodean al equipo.

La idea de que el líder debe proteger a su equipo o cubrir sus espaldas no es suficiente para asegurar el éxito del equipo. Existe una complejidad mayor, pues esta idea debe ser adoptada en todos los niveles jerárquicos. Pongamos el ejemplo de un gerente de proyectos en una empresa de TI. Este líder de proyecto es responsable de cubrirle las espaldas a su equipo subordinado inmediato, y de protegerlo de amenazas internas y externas. 
Sin embargo, hay que considerar que este líder forma parte de una jerarquía de mayor nivel, en la cual reporta a alguien jerárquicamente superior. El líder de proyecto espera que su líder (gerente PMO, por ejemplo) lo proteja de igual forma que él protege al equipo de personas que tiene a cargo. El líder del líder de proyecto espera lo mismo de su líder inmediato superior y así sucesivamente. De esta forma se crea una red de equipos, los cuales pertenecen todos a una tribu más grande, la empresa.

La figura 3 ilustra esta analogía. En la izquierda podemos visualizar al líder de un equipo que delimita su círculo de protección en el cual incluye a su equipo subordinado inmediato, y en la derecha observamos ese mismo equipo formando parte de toda la jerarquía de la empresa.

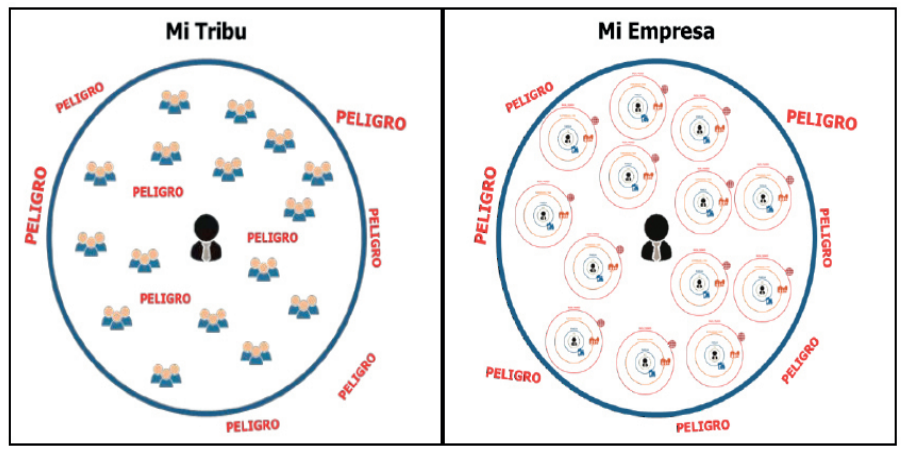

Figura 3. Límite de un equipo y límite organizacional

Elaboración propia

La jerarquía de protección descrita, más el concepto de sacrificio esperado por parte de los líderes, es la pieza restante para comprender qué está faltando en el ámbito organizacional para generar el tipo de equipos que deseamos. Veamos nuevamente la figura 3. A nivel de empresa existirán limites organizacionales, es decir, aquellas líneas definidas que separan a aquellos que pertenecen a la empresa de aquellos que no. Los colaboradores que se encuentran en los extremos de estos límites son aquellos que tienen contacto con el mundo externo a la organización, en otras palabras, son aquellos que tratan directamente con los clientes.

\subsection{Los límites del equipo y los límites externos de la empresa}

En la medida en que el líder principal de la organización (CEO, gerente general, director) se encargue de proteger a cada miembro de su equipo directo, y cada líder dentro de esta jerarquía se haga responsable de hacer lo mismo con su equipo subordinado, los miembros más lejanos del centro de liderazgo se encargarán de realizar su trabajo de forma óptima, sin tener que preocuparse por los peligros y amenazas al equipo pues comprenderán que su líder directo los 
protegerá, y este líder directo solo podrá hacer esta tarea siempre y cuando se sienta él mismo protegido por su líder directo superior. Mientras cada miembro de la empresa se sienta protegido, su enfoque se centrará en el cliente.

No todo lo que se encuentra fuera de los límites de la organización es peligro; ahí también se encuentran los clientes y, por ende, las oportunidades de generar valor para la organización. La figura 4 ilustra esta cadena que se extiende desde el epicentro del poder de la organización hasta los límites externos de la empresa, la cual limita con los peligros externos y las oportunidades.

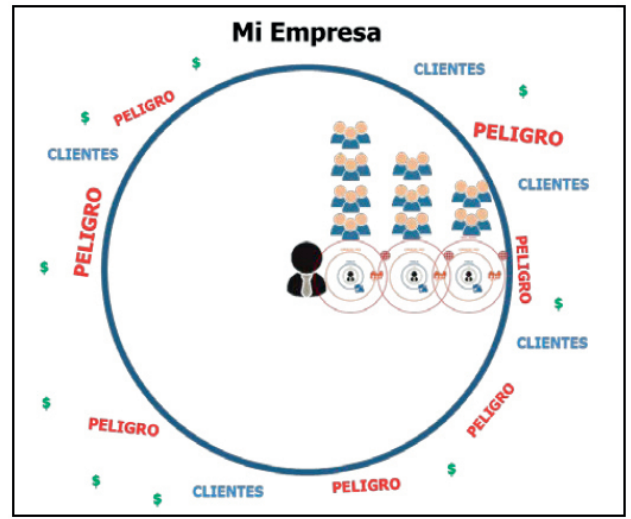

Figura 4. Límite organizacional

Elaboración propia

Kazuo Inamori, un exitoso empresario japonés, resume lo descrito anteriormente en la frase "Si quieres huevos, cuida a la gallina"

Todos los miembros del equipo esperan que su líder directo les cubra las espaldas, no que busque salvarse solo él cuando las amenazas alcanzan al equipo, o que se centre en obtener todos los beneficios del liderazgo sin pretender responder a sus obligaciones con el equipo. En la medida en que el enfoque de la organización se centre en sus colaboradores, estos cuidarán de los clientes.

Es posible afirmar que la gran mayoría de profesionales no tiene problema alguno con que sus superiores reciban una mejor paga, tengan una oficina más grande y disfruten de más y mejores beneficios otorgados por la organización. Esto responde a que, como animales sociales jerárquicos que somos, reconocemos que siempre existirá un alfa en la manda, así como un determinado lugar en la jerarquía para cada miembro. Sin embargo, esta característica social

1 “Kyocera founder's secret: Make workers happy" https://www.japantimes.co.jp/news/2015/11/05/business/ kyocera-founders-secret-make-workers-happy/\#.yHBzfi3mhry 
que arrastramos desde hace miles de años no carece de sentido. Como mencionamos anteriormente, el grupo no le otorga todos estos beneficios al alfa sin esperar algo en retorno. La tribu espera que el líder proteja a todos los miembros.

El término de protección, bajo el contexto actual de las organizaciones, se puede explicar con un ejemplo de la vida real. Barry-Wehmiller, una empresa estadounidense de tecnología se vio fuertemente impactada por la crisis financiera del 2008. Por primera vez en su carrera, el CEO tuvo que considerar el proceso de despido masivo en su compañía, algo, por cierto, muy común en Estados Unidos. Sin embargo, bajo la cultura que él mismo había impuesto, la cual tenía como fundamento el ver a cada miembro del equipo como una familia, esta acción iba en contra de los principios organizacionales establecidos. Robert H. Chapman, el CEO de BarryWehmiller, tomo una medida poco convencional que ejemplifica el significado de proteger que el presente artículo pretende explicar.

Chapman implementó un programa obligatorio de licencia sin goce de haber; es decir, todos (incluido el CEO) en la compañía saldrían de vacaciones sin paga alguna. De esta forma se reducía el gasto en planilla y la empresa podría mantenerse a flote durante la crisis financiera. La lógica de Chapman era la siguiente. Es mejor que todos sufran un poco, a que unos cuantos sufran mucho. Tal vez lo primero que se nos vendría a la mente seria que hubo una serie de reclamos en todos los diferentes niveles de la organización por parte de aquellos que no deseaban sacrificarse por otros, especialmente aquellos que se sentían seguros de quedar fuera de la lista del despido masivo que se veía inminente. Sin embargo, este no fue el caso. Todo lo contrario, hubo colaboradores dispuestos a salir de licencia más tiempo, con tal de apoyar a aquellos que no podían dejar de recibir un sueldo completo por un mes. Todos los miembros de Barry-Wehmiller estuvieron de acuerdo con la política. Aquellos que no podían soportar el impacto financiero de salir un mes de licencia sin goce de haber, se vieron apoyados por aquellos que si podían soportarlo financieramente. Nadie fue dejado de lado y nadie fue despedido. Posteriormente, la crisis se asentó y Barry-Wehmiller continúa a la fecha operando (Sinek, 2014).

Esto es un reflejo de la cultura impuesta por el líder supremo de Barry-Wehmiller, que consideraba la protección de cada miembro del equipo como algo fundamental, lo cual ejemplificaba la cultura organizacional. Chapman lo ponía de la siguiente forma: no puedes despedir a tus propios hijos.

El líder pone los intereses de su equipo antes que los de él mismo, ya sea que se trate de aminorar un impacto negativo grande como un despido masivo, o que un área externa o cliente comience a amedrentar a un miembro del equipo. El líder se encargará de poner el pecho para él mismo recibir los golpes de las amenazas antes que dejar que alguien de su equipo sufra. 


\subsection{Las generaciones y el agile mindset}

Existe una problemática muy de moda hoy en día en las organizaciones. La diferencia de edad de los miembros de un equipo o, puesto de otra forma, la interacción y roces entre las diferentes generaciones (boomers, gen X, millennials) que coexisten en una organización.

En la gran mayoría de organizaciones existirá resistencia al cambio cuando se trata de un cambio cultural de alto impacto, como es pasar de una gestión de proyectos tradicional a una gestión de proyectos ágiles. Para que una gestión de proyectos ágiles funcione de forma óptima, la organización debe asimilar el mindset de forma vertical, desde el CEO y el directorio hasta el miembro más junior del equipo. Este cambio, usualmente disruptivo para muchos, tiene casi siempre como barrera adicional el choque generacional. Las generaciones más jóvenes, millennials y generación $\mathrm{X}$, tienden a asimilar mejor el agile mindset $\mathrm{y}$ sus implicancias de cambios.

Considerando que las generaciones más antiguas (baby boomers) se encuentran en puestos jerárquicos superiores, el entrenamiento relacionado al agile mindset debe ser más abstracto y orientado a beneficios que técnico o de procesos, como lo sería para el mid-management. Es imperativo iniciar la capacitación del personal desde la parte superior de la pirámide organizacional, orientándolo a dar a entender los beneficios que se obtendrían al aplicar el agile mindset en la organización, para posteriormente pasar a la capacitación del personal de mid-management y de primera línea, que serán los que participarán y ejecutarán los proyectos, programas y portafolios.

\subsection{El agile mindset, el liderazgo y la motivación}

Los conceptos, teorías e ideas descritas en este artículo no son ninguna novedad. Los autores del Manifiesto ágil identificaron el enfoque en el equipo dentro de sus valores y principios ágiles.

Sin embargo, hacía falta comprender aquellos aspectos ocultos del liderazgo y la motivación para poder entender por qué ciertas técnicas o prácticas de motivación y liderazgo fallaban.

Todo está relacionado con las necesidades de los miembros de un equipo y el tipo de liderazgo que tienen. La figura 5 muestra esta cadena. 


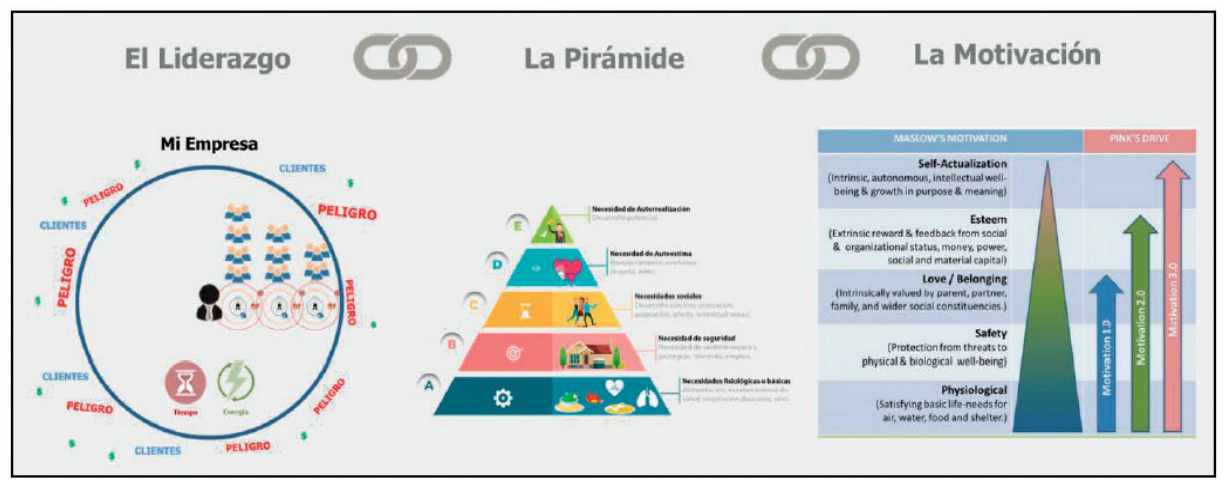

Figura 5. Relación entre el círculo de protección del liderazgo, la pirámide de necesidades de Maslow y las etapas de la evolución de la motivación

Elaboración propia

Con el alineamiento del agile mindset a los aspectos ocultos de la motivación y el liderazgo podemos entender por qué se le da suma importancia al aspecto humano en la gestión de proyectos ágiles. Un equipo de alto desempeño se define como un grupo de personas con roles específicos y talentos y habilidades complementarias que están alineados y comprometidos con un propósito común, y que consistentemente demuestran altos niveles de colaboración e innovación para producir resultados superiores.

No es posible tener un equipo con alta colaboración e innovación si sus miembros no logran alcanzar el último nivel de la pirámide de Maslow (1943), la autorrealización, que es donde se dan la creatividad, la espontaneidad y la resolución de problemas. Es imperativo además comprender la relación directa del nivel de autorrealización con la teoría de la motivación 3.0 de Pink (2011). Sin embargo, por más que se logre alcanzar estos altos niveles de satisfacción entre los miembros de un equipo, el desempeño a largo plazo solo puede ser asegurado por un liderazgo adecuado, en el cual el líder tenga claras sus responsabilidades y el costo que implica ostentar dicho título.

La aplicación de los métodos, marcos de trabajo y buenas prácticas de gestión de proyectos y personas, junto con la comprensión e interiorización de los conceptos e ideas presentadas en este artículo, pueden facilitar, de forma consistente, la generación de equipos de alto desempeño a nivel de proyecto y organización. 


\section{Discusión de CaSOS EMPRESARIALES: NeXT Jump Y ETERNIASOFT}

Para ilustrar el significado de estos conceptos tomaremos dos ejemplos de ambientes empresariales reales. El primero es de la empresa Next Jump, una compañía estadounidense de e-commerce que nació en el dormitorio de la universidad Tufts en 1994 por iniciativa de su fundador, Charlie Kim.

Para el 2012, Next Jump era ya considerada una empresa exitosa financieramente. Aunque relativamente pequeña en tamaño, su solvencia económica y prospectivas futuras eran sólidas.

Sin embargo, en ese mismo año, su fundador tuvo una muy productiva reunión con el CEO de Barry-Wehmiller, Robert H. Chapman, el mismo que evitó el despido masivo en Barry- Wehmiller. A esta reunión también asistió el exitoso orador de liderazgo, Simon Sinek. En esta reunión Charlie Kim toma la drástica decisión de realizar un cambio organizacional de alto impacto. Es así como, en abril del 2012, Next Jump implementa su política de No Firing Policy (Política de No Despido) (Sinek, 2014).

Esta política, además de incrementar los estándares de contratación de personal para la compañía, indicaba explícitamente que a ninguno de sus colaboradores se le podría despedir bajo el pretexto de balancear los libros contables (para reducir costos), errores operativos costosos o bajo rendimiento. La política indicaba que la empresa invertiría tiempo y recursos para identificar la problemática de fondo y ayudar a las personas a superar cualquier obstáculo que impidiera su óptimo desempeño. Las únicas situaciones donde un colaborador podría ser despedido serían si este realizaba acciones fuera al ámbito moral y de los valores de la compañía o si sus acciones atentaban directa y deliberadamente contra sus colegas.

Esta política implantada por el fundador le valió a Next Jump incrementar su rentabilidad de $12 \%$ a $60 \%$ anual en tan solo un año. Incluso el personal que no temía ser despedido por tener un histórico de alto rendimiento notó la diferencia al verse rodeado de colegas cuyo desempeño y motivación aumentaron drásticamente, lo cual generó un incremento en la calidad del clima laboral organizacional y, por ende, un incremento en el desempeño y la rentabilidad de la organización.

En el 2016, Next Jump fue seleccionada como una de las tres compañías que ostentan el título de deliberately developmental organisation (organización deliberadamente evolutiva), título otorgado por Robert Kegan en su libro An Everyone Culture: Becoming a Deliberately Developmental Organization, publicado por Harvard Business Review; un reconocimiento otorgado por el excelente clima laboral que tiene Next Jump desde la implementación de su política de no despidos.

Charlie Kim siguió un principio muy básico, el de proteger a sus colaboradores como si fueran sus hijos (dijo esto literalmente). Y aunque esto pueda sonar muy poético, si se 
implementa a un nivel cultural que afecte verticalmente a la organización, los resultados serán bastante tangibles.

La política generó que los colaboradores se sintieran protegidos e incluidos en la organización independientemente de cuál fuera su puesto, y esto responde a la lógica de que un miembro de un equipo está mejor equipado para aprovechar las oportunidades y afrontar las amenazas externas cuando no percibe amenaza alguna de sus compañeros al interior de la organización.

No es posible exigirles a las personas que confíen, que tengan grandes ideas innovadoras y ciertamente tampoco que cooperen. Estos son siempre resultados, los resultados de que estas mismas personas se sientan seguras y en confianza con los demás miembros del equipo con quienes trabajan y la certeza de que su líder inmediato está ahí para motivarlos y ayudarlos a crecer en vez de amenazarlos con represalias de despido.

El segundo caso de éxito es sobre una empresa de origen peruano llamada Eterniasoft, compañía de tecnología que por el 2013 desarrolló el software Sperant, un CRM inmobiliario que actualmente ostenta el mayor nivel de participación de mercado en su rubro.

Su crecimiento vertiginoso desde el 2013, con cero clientes, a la actualidad con más de 60 clientes, no se debió a una gran inversión inicial ni tampoco a las habilidades de programación (promedio) de uno de los fundadores, y ciertamente tampoco a sus oficinas ubicadas en el distrito de San Borja, lejos de los lujos del corazón financiero peruano; ni qué hablar de los sueldos que ha tenido y tiene aún su personal, por debajo del promedio del mercado. Este crecimiento fuera de precedentes se debió a la calidad del software que desarrolló la empresa, el cual cumplía con todas las expectativas (y más) de sus clientes. Adicionalmente, las políticas y procesos posventa, enfocadas en la calidad de atención al cliente, le valieron mantener hasta la fecha a cada uno de los clientes que adquirieron al largo de los años. No solo lograban vender el acceso a su CRM online, sino que lograban también retener, a través una óptima atención posventa, a todos los clientes que entraban en su red.

Sin embargo, para lograr todo lo descrito anteriormente, Eterniasoft no contrató grandes cantidades de programadores, analistas funcionales, asistentes posventa o genios comerciales. De hecho, hasta ahora mantienen un equipo relativamente pequeño, con 14 colaboradores más los dos socios fundadores. La calidad ofrecida en el software y el servicio posventa se vieron soportados por el alto desempeño del equipo, lo cual a su vez se vio soportado por el tipo de cultura organizacional que se sembró en un inicio.

Gonzalo Ramírez, exalumno de la Universidad de Lima y gerente de Desarrollo de Eterniasoft, mantiene hasta la fecha un tipo de liderazgo servicial, inclusivo, el cual se preocupa por que cada miembro de su equipo se encuentre enfocado y cómodo con las funciones que desempeña. No es necesario leer indicadores de desempeño, basta con ir a sus oficinas y darse cuenta de que absolutamente todos sus colaboradores gozan de una flexibilidad de horario de 
la que muy pocas empresas peruanas pueden jactarse. Llegar a las $10 \mathrm{u} 11$ de la mañana es algo que pocos pueden hacer. Recibir un préstamo directo de su empresa para financiar un departamento propio es algo impensable para muchos. Tener almuerzos pagados todos los meses, una fiesta de aniversario y una fiesta de fin de año para generar integración es el estándar para todos los miembros de Eterniasoft. Pero lo más destacable es que todos ellos alcanzan ese nivel de desempeño por la simple razón que se sienten protegidos, incluidos y pueden ver que la cantidad de horas (de más) que le dedican a la empresa es algo que incluso sus propios líderes hacen, por lo que es más fácil seguir el ejemplo. Como costumbre bomberil de su gerente de Desarrollo (quien es bombero voluntario), él suele aplicar lo que llama "Ojo al guía”, es decir, que el líder (guía) debe primero demostrar que puede hacerlo y luego puede exigir a su equipo que lo haga igual o mejor.

Saber que cualquier miembro del equipo responderá el teléfono a las nueve o 10 de la noche para atender un requerimiento de urgencia y, sobre todo, que lo hará con empeño y sin titubear, demuestra el nivel de involucramiento del personal. Eterniasoft sabe hacer sentir incluidos a sus colaboradores, protegidos de peligros externos y lo hace sin establecer políticas estrictas de horarios laborales o penalidades por bajo desempeño, lo hace cuidando de ellos. Como respuesta a este cuidado, cada miembro del equipo de Eterniasoft se compromete profundamente con la calidad de atención que les brindan a sus clientes. Su enfoque no está en lograr el nivel de ventas esperadas, ni en completar la mayor cantidad de requerimientos. Su enfoque está en los clientes, en mantenerlos satisfechos y cuidar de sus necesidades.

Eterniasoft cuida a sus colaboradores y estos, en respuesta, cuidan a los clientes. Este nivel de integración y protección es el mismo que utilizaron Robert H. Chapman y Charlie Kim en sus respectivas organizaciones; aquel que ve a cada miembro de su empresa como miembros de una familia y se preocupa por protegerlos.

Los ejemplos descritos pueden resumirse en el siguiente extracto del libro de Sinek (2014):

- Los líderes son aquellos que corren primero hacia lo desconocido. Ellos se apresuran hacia el peligro.

- Ellos ponen sus propios intereses a un lado para protegernos o para jalarnos hacia el futuro.

- Los líderes primero sacrificarían lo que es suyo para salvar lo que es nuestro y jamás sacrificarían lo que es nuestro para salvar lo que es suyo.

- Esto es lo que significa ser un líder: que ellos escogen ir primero hacia el peligro, ir primero hacia lo desconocido.

- Y cuando nos sentimos seguros de que nos mantendrán a salvo, marchamos detrás de ellos y trabajamos sin cansancio para ver su visión cobrar vida y orgullosamente llamarnos sus "seguidores". 


\section{RETOS DE LOS EQUIPOS DE ALTO RENDIMIENTO}

\subsection{El liderazgo y la falta de habilidades}

Las dos empresas tomadas como ejemplo son organizaciones ya establecidas que lograron, a través de una cultura organizacional óptima ya establecida, sacar lo mejor de sus colaboradores. Sin embargo, quedan aún algunas preguntas abiertas respecto a las situaciones que resultan de diversos contextos en los cuales los equipos de trabajo interactúan. Por ejemplo, ¿cómo se ven afectadas la motivación y el liderazgo cuando se trata de un equipo de proyecto sin las skills o la seniority, necesarias para ejecutar el proyecto asignado? $\mathrm{O}$ en una situación similar, cuando el equipo está muy motivado pero deben manejar una tecnología nueva que nadie del equipo domina.

Tomaremos el caso de Eterniasoft descrito párrafos arriba. En sus inicios Eterniasoft no contaba con fondos para invertir en un equipo de desarrollo de software de alto desempeño. De hecho, los primeros dos desarrolladores que se incorporaron en un inicio y que a la fecha siguen con la empresa vienen de la ciudad de Cerro de Pasco, de donde decidieron migrar hacia Lima en busca de mejores oportunidades laborales. Sus habilidades de programación apenas alcanzaban el nivel de un programador middle. Incluso la única experiencia que poseían era la de trabajar en los laboratorios de su universidad local.

Es innegable que las habilidades blandas son bastante valiosas para el trabajo en equipo, pero las habilidades duras o conocimientos técnicos y conceptuales, son la masilla que usa el líder para crear la escultura. Al carecer de este insumo, el líder tiene un reto mayor, el cual no es solo motivar a un equipo que carece de los suficientes conocimientos técnicos, sino que debe empezar haciendo que el mismo equipo cree su propia masilla (habilidades técnicas) y con el tiempo, el propio equipo impulsará su hambre de conocimiento. La falta de habilidades o skills es un reto para el líder.

El reto del líder con un equipo que no cuenta con las skills suficientes está primero en asegurar la motivación del equipo para adquirir las habilidades que les faltan. En el caso de Eterniasoft, el desarrollo del primer beta del CRM inmobiliario que crearon demoró aproximadamente un año en salir al mercado. Los fundadores hicieron una inversión, una apuesta por el equipo y sus habilidades. Apostaron por ellos y asumieron el riesgo de desarrollar un equipo para crear un CRM que ahora es líder en el mercado peruano y que ya cuenta con presencia en otros países de Latinoamérica como Ecuador y México.

Un aspecto bastante relacionado al liderazgo y la motivación es el concepto de $100 \%$ responsable. Este concepto indica que cada persona es responsable absoluta de su propia realidad y en este caso particular, hablando de los líderes, estos son responsables del desempeño de cada miembro. 
El líder debe ser capaz de identificar las debilidades de los miembros de su equipo para que así, de forma proactiva, se resuelvan, y su desempeño no se vea afectado. El líder no solo es responsable de motivar, sino que cuando incluimos la variable de $100 \%$ responsable, es necesario comprender que, si alguien en el equipo no está rindiendo como debe, es el líder el que debe encargarse de su desarrollo y crecimiento. Es el líder quien debe identificar la forma óptima de explotar las habilidades de cada miembro.

Si el líder no es capaz de llegar a la persona, encontrar lo que la motiva o resolver el conflicto o impedimento para que se desempeñe de forma óptima, entonces se trata de la incapacidad del mismo líder de resolver o superar lo que está impidiendo que aquel miembro de su equipo alcance un desempeño óptimo. Por supuesto, esto parte del supuesto de que la persona que es parte del equipo ha pasado por los filtros necesarios de reclutamiento y selección y cumple con los requerimientos mínimos indispensables para cumplir sus funciones. Si aquel miembro no rinde, es el líder quien debe responder y encontrar una solución apropiada; como dice Jocko Willink, SEAL de la Armada estadounidense en retiro: "No hay equipos malos; solo líderes malos”2.

\subsection{Cuando se impone a un líder}

Una situación común en muchas organizaciones es la imposición de una persona en un puesto de autoridad, lo que impacta en el clima laboral de la organización de diversas maneras, ya sea que el nuevo líder impuesto tenga una reputación conocida o que sus valores no estén del todo alineados con la organización pero logró obtener el título debido a otras variables. Un ejemplo que describe esta situación es el caso de la empresa Fritz Publishing.

Fritz Publishing fue hasta el 2009 la empresa de publicidad más respetada y reconocida en el continente africano. Con casi 50 años de trayectoria, la empresa gozaba de un excelente desempeño debido a su cultura de empoderamiento, inclusión y capacitación de sus 135 colaboradores (Ronnie, 2017).

En el 2009, cuando la familia fundadora decide vender el negocio, un grupo alemán, Prys Group, adquiere la compañía, haciendo un giro de 180 grados en la organización y su cultura. Con un nuevo CEO, cuyas credenciales académicas y logros profesionales convencerían a muchos, Fritz Publishing entré en una nueva era de liderazgo. Sin embargo, y a pesar de que todos los colaboradores decidieron darle una oportunidad al nuevo CEO, el cambio generado no fue para bien.

2 TODAY'S IDEA: No bad teams, only bad leaders (https://entregurus.com/no-bad-teams-only-bad-leaders). 
El ruso Vadim Arshavin tomó las riendas del negocio. El flamante nuevo CEO era conocido por tener un liderazgo duro y enfocado principalmente en los números; no en la gente. Esto traería como consecuencia que la ratio de renuncia de la empresa se disparara a 17,2 \%, algo que jamás había sucedido en la historia de la compañía. Adicionalmente el trato con los colaboradores cambió. Si a alguien no les gustaba el nuevo ambiente orientado a resultados, y que dejaba de lado el interés por las personas, era explícitamente invitado a buscar nuevas oportunidades laborales en el mercado.

La contratación de la esposa del nuevo CEO a los 18 meses de iniciado su reinado en la empresa tampoco fue una señal del todo adecuada. Saltándose los procesos formales de contratación, esto tuvo un impacto bastante negativo en los altos ejecutivos (de antes del cambio de liderazgo) que aún permanecían con la compañía. Sumado a esto, el Plan de Equidad Laboral (Employment Equity Plan) que Fritz había puesto en marcha años atrás para mantener un equilibrio étnico y cultural dentro de la organización, había sido dejado de lado, lo que ocasionaba que la diversidad étnica original de la empresa se redujera drásticamente.

Como resultado, la moral entre los colaboradores se vio drásticamente disminuida debido a los cambios forzosos que se dieron. La clientela comenzó a notar que los empleados de Fritz, con quienes habían trabajado por años y mantenían contacto directo, se encontraban más desenganchados con el sentido de urgencia de la clientela, que no estaban enfocados en sus funciones y que más bien parecían dedicar más tiempo y energía a otras cosas que no eran la atención y satisfacción del cliente. Ciertamente una imagen muy lejana de la impecable reputación que Fritz había ostentado por varias décadas.

A enero del 2016, Fritz Publishing había dejado de ser la compañía emblemática que una vez fue, donde los colaboradores se sentían protegidos, valorados e incluidos, y donde, a cambio, estos cuidaban a la clientela. Los clientes habían empezado a fugarse de forma masiva hacia la competencia, argumentando que la calidad del servicio había sufrido un revés en los últimos años.

La problemática que se aprecia fue el cambio cultural orientado a los números y no a las personas. Cuando el líder de una organización tiene en su cabeza a los números antes que a los seres humanos que dirige, la cultura organizacional se ve afectada pues la pirámide de la empresa funciona como un tobogán, donde aquel pensamiento fluye desde lo más alto hacia los niveles inferiores de la empresa. Cuando el líder pasa de enfocarse en sus colaboradores a enfocarse en los números, aquella forma de pensar y actuar también se transmite a todos los miembros del equipo.

Esto usualmente se traduce en competencia por comisiones, largas horas de trabajo, enfoque en llegar a la meta, lo cual a su vez resta el tiempo y energía que les dedican a los clientes para enfocarlo en no solo cubrir sus espaldas, sino alcanzar un número a costa de otros. 


\section{Conclusiones}

En el caso de las organizaciones que deseen incorporar metodologías ágiles para la gestión de sus proyectos será necesario primero realizar un análisis de cómo su cultura organizacional se podrá adaptar a los valores y principios ágiles. Si esto no es posible y no es posible establecer un agile mindset a lo largo de la organización con su cultura actual, de nada servirá la inversión de tiempo y recursos. La alternativa será un cambio cultural para adaptarse a las metodologías ágiles.

El actual modelo motivacional 2.0 que las empresas tienen necesita empezar a migrar al modelo 3.0, el cual facilitará la generación de equipos de alto desempeño. Sin embargo, se debe considerar que este es un cambio de cultura organizacional de alto impacto y debe realizarse con los procesos y personas adecuadas.

Los equipos de alto desempeño no se crean al azar. Es necesario un apropiado entendimiento de la dinámica de que existe entre la motivación, el liderazgo y la cultura organizacional para identificar cómo crear un ambiente adecuado para alcanzar un desempeño superior.

La aplicación del concepto del círculo de protección del liderazgo no debe reducirse al nivel de proyecto, sino que debe aplicarse a nivel organizacional para que funcione de forma transversal en todas las áreas. Este concepto debe adaptarse y estar embebido en la cultura organizacional si se desea obtener los beneficios identificados y descritos en este artículo.

\section{REFERENCIAS}

Maslow, A. (1943). A theory of human motivation. Washington DC: Psychological Review. American Psychological Association.

Pink, D. H. (2011). Drive: the surprising truth about what motivates us. Nueva York: Riverhead Books.

Ronnie, L. (2017). Leadership challenges at Fritz Publishing. Emerald Emerging Markets Case Studies, 7(3), pp. 1-17.

Sinek, S. (2014). Leaders Eat Last. Nueva York: Penguin Group. 


\section{Bibliografía}

Knaster, R., y Leffingwell, D. (2017). SAFe 4.0 Distilled: Applying the Scaled Agile Framework for Lean Software and Systems Engineering. Addison-Wesley Professional.

Project Management Institute (2017). A guide to the project management body of knowledge. PMBOK guide (sexta edición). Pennsylvania: Project Management Institute.

Project Management Institute (2017). Agile practice guide. Pennsylvania: Project Management Institute.

Robins, S. P., y Timothy Judge, T. A. (2013). Organizational Behavior (15.a edición). Nueva Jersey: Pearson Education.

SCRUM Study (2017). A Guide to the Scrum Body of Knowledge (SBOK ${ }^{\mathrm{mw}}$ Guide) (3. ${ }^{\mathrm{a}}$ edición). Arizona: VMEdu. 
